\title{
Feasibility study of simultaneous multislice diffusion kurtosis imaging with different acceleration factors in the liver
}

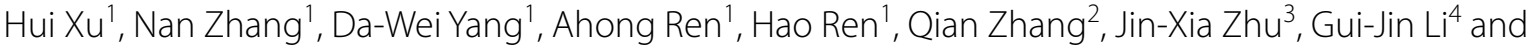 \\ Zheng-Han Yang ${ }^{1^{*}}$
}

\begin{abstract}
Background: Simultaneous multislice diffusion-weighted imaging (SMS-DWI) has been used to reduce image acquisition time. The purpose of this study was to investigate the feasibility of diffusion kurtosis imaging (DKI) based on the SMS technique in the liver and the influence of this method compared with that of conventional DWI sequences on image quality and DKI-derived quantitative parameters.

Methods: Forty volunteers underwent SMS-DWI sequences with acceleration factors of 2 and 3 (SMS2-DWI, SMS3DWI) and conventional DWI (C-DWI) of the liver with three b-values $\left(50,800,2000 \mathrm{~s} / \mathrm{mm}^{2}\right)$ in a 3 T system. Qualitative image quality parameters and quantitative measurements of the signal-to-noise ratio (SNR), mean kurtosis (MK), mean apparent diffusivity (MD) and apparent diffusion coefficient (ADC) for the liver were compared between the three sequences.
\end{abstract}

Results: The scan times of C-DWI, SMS2-DWI, and SMS3-DWI were 4 min 11 s, 2 min 2 s, and 1 min 34 s, respectively. For all image quality parameters, there were no significant differences observed between C-DWI and SMS2-DWI (all $p>0.05$ ) in the images with b-values of 800 and $2000 \mathrm{~s} / \mathrm{mm}^{2}$. C-DWI and SMS2-DWI exhibited better scores than SMS3-DWI (all $p<0.01$ ) in the images with b-values of $2000 \mathrm{~s} / \mathrm{mm}^{2}$. In the images with b-values of $800 \mathrm{~s} / \mathrm{mm}^{2}, C$-DWI and SMS2-DWI exhibited better scores than SMS3-DWI for artefacts and overall image quality (all $p<0.01$ ), and C-DWI exhibited better scores than SMS3-DWI for the visibility of intrahepatic vessels $(p<0.001)$. There were no significant differences in the sharpness of the right lobe edge $(p=0.144)$, conspicuity of the left lobe $(p=0.370)$ or visibility of intrahepatic vessels $(p=0.109)$ between SMS2-DWI and SMS3-DWI. There were no significant differences in the sharpness of the right lobe edge $(p=0.066)$ or conspicuity of the left lobe $(p=0.131)$ between C-DWI and SMS3DWI. For the b-value of $800 \mathrm{~s} / \mathrm{mm}^{2}$, there were no statistically significant differences between SMS2-DWI and C-DWI $(p=1.000)$ or between SMS2-DWI and SMS3-DWI $(p=0.059)$, whereas SMS3-DWI had a significantly lower SNR than C-DWI $(p=0.024)$. For the DKI-derived parameters (MK and MD) and ADC values, there were no significant differences between the three sequences ( $M K, p=0.606 ; \mathrm{MD}, p=0.831 ; \mathrm{ADC}, p=0.264$ ).

Conclusions: SMS-DWI with an acceleration factor of 2 is feasible for the liver, resulting in considerable reductions in scan time while maintaining similar image quality, comparable DKI parameters and ADC values compared with those of C-DWI.

Keywords: Diffusion kurtosis imaging, Simultaneous multislice, Liver, Magnetic resonance imaging

\footnotetext{
*Correspondence: yangzhenghan@vip.163.com

1 Department of Radiology, Beijing Friendship Hospital, Capital Medical

University, 95 Yong an Road, Xicheng District, Beijing 100050, China

Full list of author information is available at the end of the article
}

(C) The Author(s) 2021. Open Access This article is licensed under a Creative Commons Attribution 4.0 International License, which permits use, sharing, adaptation, distribution and reproduction in any medium or format, as long as you give appropriate credit to the original author(s) and the source, provide a link to the Creative Commons licence, and indicate if changes were made. The images or other third party material in this article are included in the article's Creative Commons licence, unless indicated otherwise in a credit line to the material. If material is not included in the article's Creative Commons licence and your intended use is not permitted by statutory regulation or exceeds the permitted use, you will need to obtain permission directly from the copyright holder. To view a copy of this licence, visit http://creativecommons.org/licenses/by/4.0/. The Creative Commons Public Domain Dedication waiver (http://creativecommons.org/publicdomain/zero/1.0/) applies to the data made available in this article, unless otherwise stated in a credit line to the data. 


\section{Background}

Diffusion kurtosis imaging (DKI), first proposed by Jensen et al. [1], has been widely used to evaluate the degree of liver fibrosis, tumour characteristics, and treatment responses [2-4]. This imaging method requires ultrahigh b-values $\left(1500-2000 \mathrm{~s} / \mathrm{mm}^{2}\right)$ to facilitate the successful capture of non-Gaussian behaviour $[5,6]$. However, ultrahigh b-value DWI results in a decrease in the signal-to-noise ratio (SNR) and an increase in anatomic distortions. To obtain good image quality, increasing the average time for ultrahigh b-values is essential and leads to relatively long scan times. Therefore, the technique of reducing the scan time is very important for promoting the routine application of DKI sequences for the liver.

Recently, simultaneous multislice DWI (SMS-DWI) has been used to reduce the image acquisition time [7-10]; SMS-DWI is based on the excitation of multiple slices simultaneously and subsequent antialiasing to reconstruct the magnitude images. Slices are separated using information about coil sensitivities from phased array coils, gradients, or radiofrequency encoding. The SMS technique shortens the scan time by increasing the number of acceleration factors (AFs), and it was initially used in the brain [11]. In recent years, several studies have reported that the SMS technique shortens the scan time of DWI in the liver, pancreas, prostate, rectum, breast, and kidney [12-16]. A limited number of studies have focused on intravoxel incoherent motion (IVIM) analysis based on SMS-DWI sequences and found a significant scan time reduction [13, 17]. Phi Van et al. reported slight systematic deviations in the IVIM parameters with increased AFs [17]. However, no studies have examined the impact of DKI parameter calculations based on SMS techniques in the liver.

Therefore, the purpose of our study was to investigate the feasibility of DKI based on the SMS technique in the liver and the influence of this method compared with conventional DWI sequences on image quality and DKI-derived quantitative parameters.

\section{Methods \\ Subjects}

A total of 40 volunteers ( 23 females and 17 males; age range $24-53$ years, mean 37 years) without a known history of alcohol or drug abuse, viral hepatitis, or prior liver surgery were enrolled.

\section{DWI protocols}

All DWI data were acquired on a 3.0T whole-body MRI scanner (MAGNETOM Prisma, Siemens Healthcare, Erlangen, Germany). An 18-channel phased-arrayed body coil in combination with 12 elements of a 32-channel spine coil was used.

In all the participants, SMS-DWI sequences with acceleration factors of 2 and 3 (SMS2-DWI and SMS3DWI, respectively) and conventional DWI (C-DWI) were scanned. DWI was acquired in axial slice orientation with a slice thickness of $6 \mathrm{~mm}$ in free breathing without respiratory compensation techniques such as respiratory triggering and navigator triggering. Diffusion gradients were applied in four-scan traces with b-values of 50, 800 and $2000 \mathrm{~s} / \mathrm{mm}^{2}$. Fat suppression with a spectral attenuated inversion recovery (SPAIR) technique was used. Table 1 provides detailed sequence parameters, including scan time.

\section{Image assessment}

\section{Qualitative analysis of image quality}

All the images were transferred to a workstation (Syngo. via, VB10; Siemens Healthcare, Erlangen, Germany).

The quality of the images with b-values of $800 \mathrm{~s} / \mathrm{mm}^{2}$ and $2000 \mathrm{~s} / \mathrm{mm}^{2}$ was independently evaluated by two radiologists with 5 and 12 years of experience in liver MRI. The two radiologists were blinded to the sequence information.

A five-point Likert scale was used to rate the image quality with respect to the following aspects (sharpness of the right lobe edge, conspicuity of the left lobe, visibility of intrahepatic vessels, absence of artefacts and overall image quality), with higher scores indicating better image quality $(5=$ excellent, $4=$ good, $3=$ moderate, $2=$ poor, $1=$ not diagnostic). Regarding the occurrence of artefacts, $5=$ no artefacts, $4=$ mild artefacts, $3=$ moderate artefacts, $2=$ severe artefacts, and $1=$ severe artefacts representing no diagnosis. The image quality scores of conventional DWI, SMS2-DWI and SMS3-DWI sequences assigned by the two radiologists were recorded for each participant.

\section{Quantitative analysis of signal-to-noise}

The signal-to-noise (SNR) ratio was calculated as the ratio between the average signal intensity of the liver parenchyma and the standard deviation of the signal intensity of the air for images with b-values of 800 and $2000 \mathrm{~s} / \mathrm{mm}^{2}$ by the following formula:

$$
\mathrm{SNR}=\mathrm{SI}_{\text {liver }} / \mathrm{SD}_{\text {background }} \text {. }
$$

where $\mathrm{SI}_{\text {liver }}$ is the mean signal of the liver parenchyma and $\mathrm{SD}_{\text {background }}$ is the standard deviation of the background noise on the outside sides of the body in the phase-encoding directions. 
Table 1 Sequence parameters

\begin{tabular}{|c|c|c|c|}
\hline Scan Parameters & C-DWI & SMS2-DWI & SMS3-DWI AF3 \\
\hline Echo time, TE (ms) & 59 & 61 & 61 \\
\hline Repetition time, TR (ms) & 5200 & 2400 & 1800 \\
\hline FoV read (mm) & 420 & 420 & 420 \\
\hline FoV phase (\%) & 65.7 & 65.7 & 65.7 \\
\hline In-plane resolution $\left(\mathrm{mm}^{2}\right)$ & $1.6 \times 1.6$ & $1.6 \times 1.6$ & $1.6 \times 1.6$ \\
\hline Slice thickness (mm) & 6 & 6 & 6 \\
\hline No. slices & 30 & 30 & 30 \\
\hline Bandwidth, Hz/px & 2666 & 2488 & 2488 \\
\hline Fat saturation & SPAIR & SPAIR & SPAIR \\
\hline Shim mode & Standard & Standard & Standard \\
\hline iPAT & GRAPPA 2 & GRAPPA 2 & GRAPPA 2 \\
\hline Slice acceleration factor & None & Factor 2 & Factor 3 \\
\hline Diffusion mode & 4-scan trace & 4-scan trace & 4-scan trace \\
\hline b-values(averages) (s/mm²) & $50(1), 800(4), 2000(6)$ & $50(1), 800(4), 2000(6)$ & $50(1), 800(4), 2000(6)$ \\
\hline Acquisition time, min:s & $4: 11$ & $2: 02$ & $1: 34$ \\
\hline
\end{tabular}

C-DWI, conventional diffusion-weighted imaging; SMS2-DWI, simultaneous multislice accelerated DWI with an acceleration factor of 2; SMS3-DWI, simultaneous multislice accelerated DWI with an acceleration factor of 3; TR, time of repetition; TE, echo time; BW, bandwidth; iPAT, integrated parallel acquisition technique; GRAPPA, generalized autocalibrating partially parallel acquisition; SPAIR, spectral attenuated inversion recovery

\section{Quantitative analysis of DKI parameters and ADC values}

All the images were postprocessed using prototypic software (Body Diffusion Toolbox; Siemens Healthcare, Erlangen, Germany) to extract DKI parameter and ADC maps.

The apparent mean kurtosis (MK) maps and apparent mean diffusivity (MD) maps were calculated using all 3 b-values according to the following equation:

$$
\mathrm{S}(\mathrm{b}) / \mathrm{S}(0)=\exp \left(-\mathrm{b} \times \mathrm{MD}+\mathrm{b}^{2} \times \mathrm{MD}^{2} \times \mathrm{MK} / 6\right) .
$$

where MK is the mean kurtosis coefficient (a unitless parameter that represents the deviation of water motion from the Gaussian distribution) and $\mathrm{MD}$ is a corrected mean ADC without a Gaussian bias.

The ADC maps were calculated using all $3 \mathrm{~b}$-values with the following equation:

$$
\mathrm{S}(\mathrm{b}) / \mathrm{S}(0)=\exp (-\mathrm{b} \times \mathrm{ADC}) .
$$

Regions of interest (ROIs) were placed by a radiologist with 12 years of experience in abdominal MRI. ROIs with a diameter between 2 and $4 \mathrm{~cm}$ were manually placed in liver parenchyma segment $\mathrm{V}$ at the portal hilum level on the b $50 \mathrm{~s} / \mathrm{mm}^{2}$ images of C-DWI and then copied to the corresponding MK, MD and ADC maps of the C-DWI, SMS2-DWI and SMS3-DWI sequences at the same level and position, making sure to avoid large vessels and severe artefacts. The radiologist performed the first measurement within 2 days after the completion of the
DWI examination and then performed a second measurement 3 weeks later. Eventually, the average of all the measurements was adopted for analysis.

\section{Statistical analysis}

Statistical analyses were performed using SPSS version 26.0 software (IBM Corp., Armonk, NY USA) and MedCalc Software version 18.2.1 (MedCalc Software bvba, Mariakerke, Belgium). $P$ values of less than 0.05 were considered statistically significant.

Image quality parameters among the three DWI sequences (C-DWI, SMS2-DWI, and SMS3-DWI) were compared using the Friedman test. If the Friedman test showed statistical significance, the Dunn-Bonferroni post hoc test was performed to analyse all pairwise comparisons. The interobserver agreement on image quality was analysed by calculating linear weighted Cohen's kappa, with kappa values of 0.01-0.20 representing slight agreement, $0.21-0.40$ fair, $0.41-0.60$ moderate, $0.61-0.80$ good, and 0.81-1.00 almost perfect agreement.

The differences in the SNR, DKI-derived quantitative parameters and ADC values between the three sequences were compared using one-way ANOVA, and pairwise Bonferroni post hoc tests were performed if the ANOVA showed a significant difference. The distribution and concordance of DKI parameters and ADC values between the three sequences are shown in a Bland-Altman plot. The mean difference and $95 \%$ limits of agreement were reported. 


\section{Results}

All the sequences were successfully scanned, and the specific absorption rate (SAR) remained below individual limits in all sequences without the need for switching to the first-level mode.

\section{Scan time}

The acquisition times of C-DWI, SMS2-DWI, and SMS3-DWI were $4 \mathrm{~min} 11 \mathrm{~s}, 2 \mathrm{~min} 2 \mathrm{~s}$, and $1 \mathrm{~min} 34 \mathrm{~s}$, respectively.

Compared with C-DWI, SMS2-DWI significantly reduced the scan time by $51 \%$, and compared with C-DWI, SMS3-DWI significantly reduced the scan time by $63 \%$. Three b-value images of C-DWI, SMS2-DWI, and SMS3-DWI are shown in Figs. 1 and 2.

\section{Qualitative analysis of image quality}

The interobserver agreement of the two readers was good for image quality assessment, with $\mathrm{K}$ values of 0.714 (95\% confidence interval [CI], 0.634-0.795) for C-DWI, 0.639 (95\% CI 0.554-0.725) for SMS2-DWI, and 0.653 (95\% CI
0.579-0.726) for SMS3-DWI. The average image quality scores of the three sequences are shown in Table 2.

For all image quality parameters, there were no significant differences observed between C-DWI and SMS2DWI in all b-value images (all $p>0.05$ ) (Table 2). As shown in Figs. 1 and 2, compared with C-DWI, SMS2DWI exhibited equivalent image quality and no artefacts.

There were no significant differences between SMS2DWI and SMS3-DWI in the sharpness of the right lobe edge $(p=0.144)$, conspicuity of the left lobe $(p=0.370)$ or visibility of intrahepatic vessels $(p=0.109)$. SMS2-DWI exhibited better scores than SMS3-DWI for artefacts and overall image quality (all $p<0.01$ ) in the images with a $\mathrm{b}$-value of $800 \mathrm{~s} / \mathrm{mm}^{2}$. For the images with a b-value of $2000 \mathrm{~s} / \mathrm{mm}^{2}$, SMS2-DWI exhibited better scores than SMS3-DWI for all image quality parameters (all $p<0.01$ ).

There were no significant differences between C-DWI and SMS3-DWI in the sharpness of the right lobe edge $(p=0.066)$ or conspicuity of the left lobe $(p=0.131)$. C-DWI exhibited better scores than SMS3-DWI for the visibility of intrahepatic vessels, artefacts, and overall

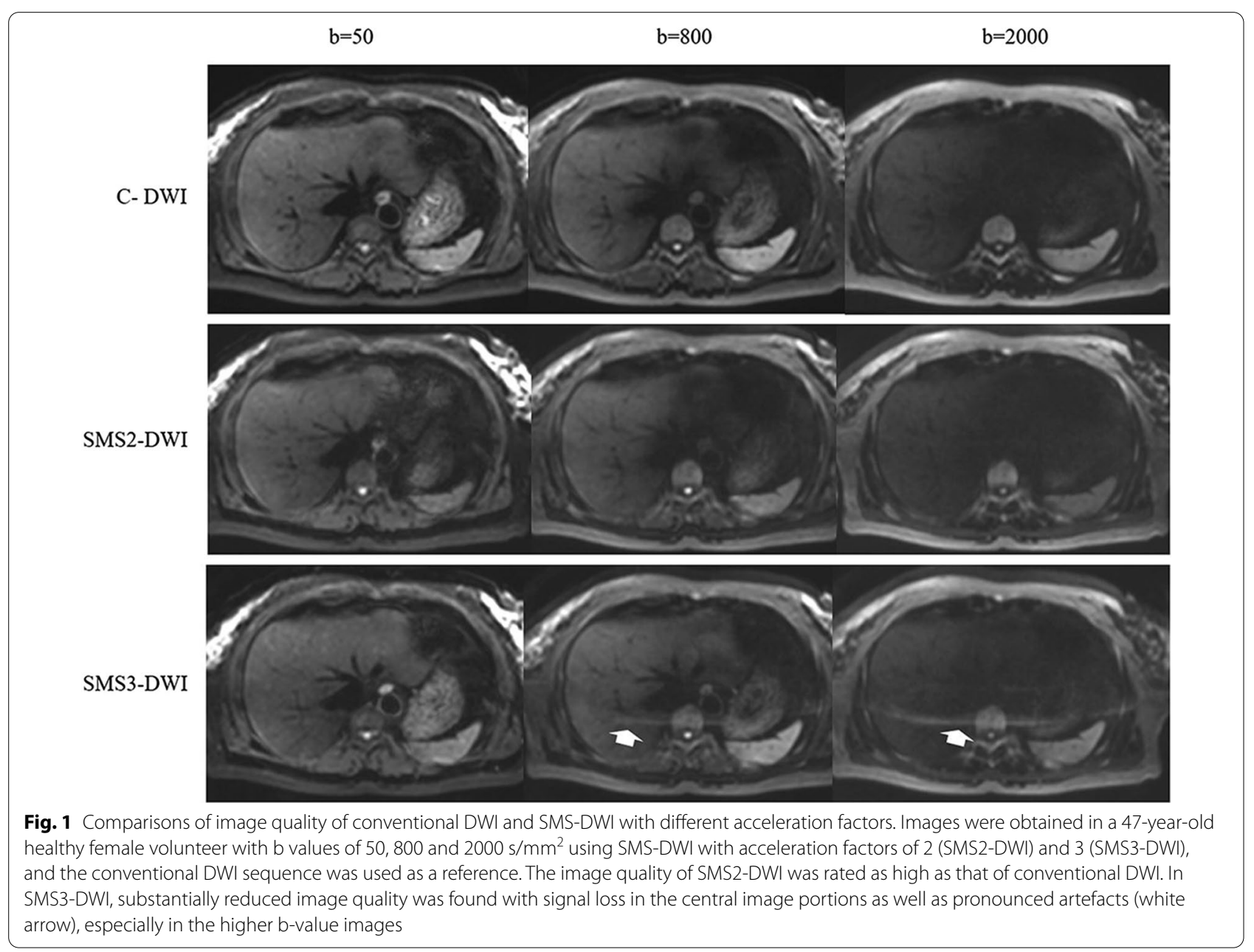




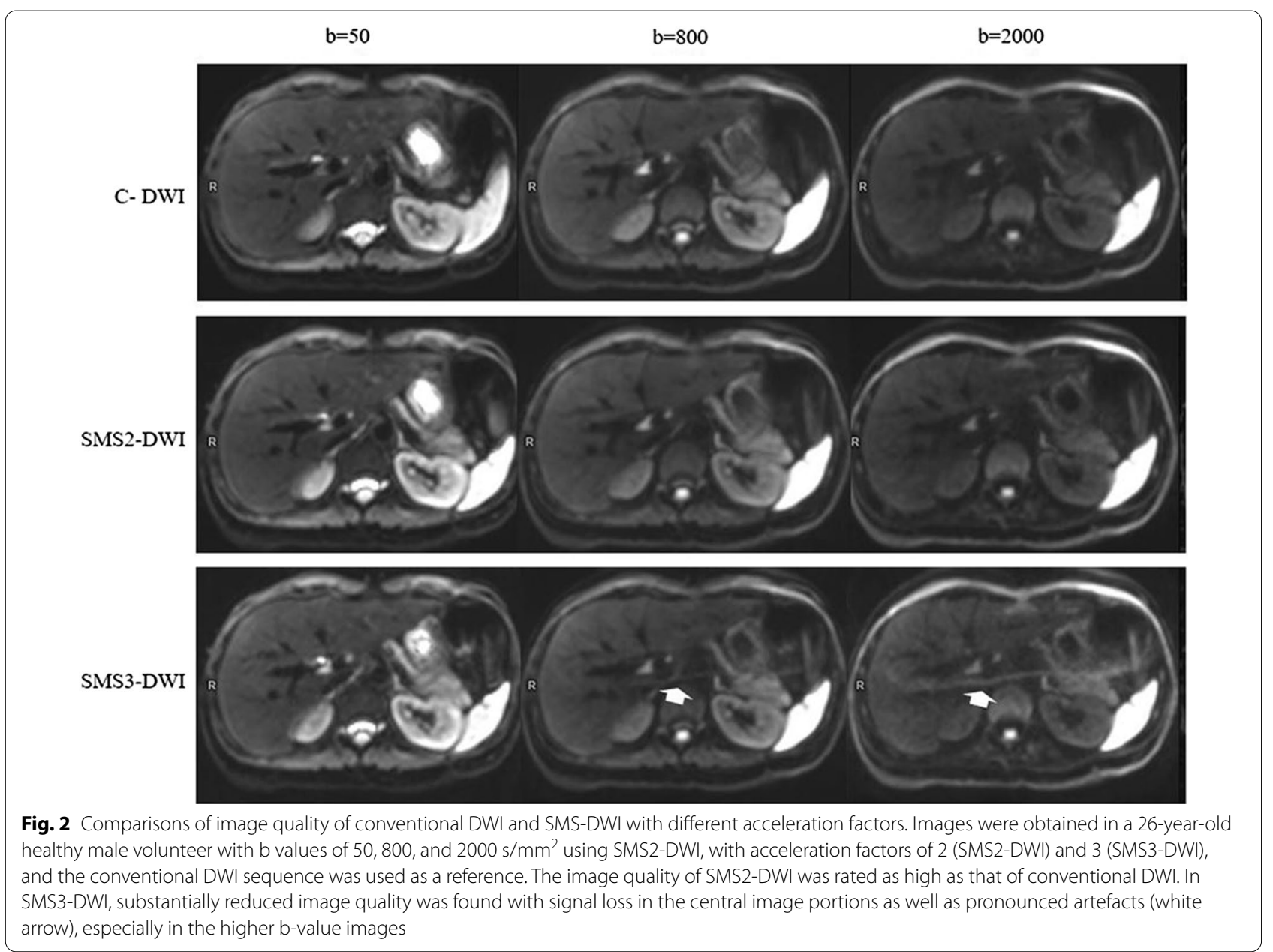

image quality (all $p<0.01$ ) in the images with a b-value of $800 \mathrm{~s} / \mathrm{mm}^{2}$. For the images with a b-value of $2000 \mathrm{~s} / \mathrm{mm}^{2}$, SMS2-DWI exhibited better scores than SMS3-DWI for all image quality parameters (all $p<0.05$ ).

Artefacts of the liver could be observed in the high b-value (800 and $2000 \mathrm{~s} / \mathrm{mm}^{2}$ ) SMS3-DWI images, and the artefacts became more obvious with increasing b-values (Figs. 1, 2).

\section{Quantitative analysis of signal-to-noise}

The SNR values measured in the three DWI sequences are shown in Table 3. There were no statistically significant differences in the SNR between the three sequences in images with a b-value of $2000 \mathrm{~s} / \mathrm{mm}^{2}(p=0.110)$. For the images with a b-value of $800 \mathrm{~s} / \mathrm{mm}^{2}$, there were no statistically significant differences between SMS2-DWI and C-DWI $(p=1.000)$ and no statistically significant differences between SMS2-DWI and SMS3-DWI $(p=0.059)$, whereas SMS3-DWI had a significantly lower SNR than C-DWI $(p=0.024)$.

\section{Quantitative analysis of DKI parameters and ADC values} The MK values of C-DWI, SMS2-DWI and SMS3-DWI were $0.67 \pm 0.15,0.68 \pm 0.11$, and $0.70 \pm 0.18$, respectively. The MD values of C-DWI, SMS2-DWI and SMS3DWI were $1.19 \pm 0.15 \times 10^{-3} \mathrm{~mm}^{2} / \mathrm{s}, 1.17 \pm 0.21 \times 10^{-3}$ $\mathrm{mm}^{2} / \mathrm{s}$, and $1.17 \pm 0.18 \times 10^{-3} \mathrm{~mm}^{2} / \mathrm{s}$, respectively. The ADC values of C-DWI, SMS2-DWI and SMS3DWI were $0.85 \pm 1.12 \times 10^{-3} \mathrm{~mm}^{2} / \mathrm{s}, 0.81 \pm 1.24 \times 10^{-3}$ $\mathrm{mm}^{2} / \mathrm{s}$ and $0.80 \pm 1.56 \times 10^{-3} \mathrm{~mm}^{2} / \mathrm{s}$, respectively. There were no statistically significant differences between the three sequences (MK, $p=0.606 ; \mathrm{MD}, p=0.831$; $\mathrm{ADC}$, $p=0.264$ ) (Fig. 3). Although there were no statistically significant differences in the DKI parameters between SMS2-DWI and C-DWI in this study, increased MK values and decreased MD values were observed with increased AFs in the liver. With an increase in the acceleration factor, the ADC values of the liver parenchyma showed a decreasing trend. The results from Bland-Altman plots are demonstrated in Fig. 4. 
Table 2 Comparison of the results of qualitative image quality scores for the three sequences

\begin{tabular}{|c|c|c|c|c|c|c|c|}
\hline Criteria & C-DWI & SMS2-DWI & SMS3-DWI & $P$ values & $\begin{array}{l}P \text { values } \\
C \text { vs. SMS2 }\end{array}$ & $\begin{array}{l}P \text { values } \\
\text { SMS2 vs. SMS3 }\end{array}$ & $\begin{array}{l}P \text { values } \\
C \text { vs. SMS3 }\end{array}$ \\
\hline \multicolumn{8}{|c|}{ Sharpness of the right lobe } \\
\hline$b=800$ & $4.88 \pm 0.40$ & $4.84 \pm 0.46$ & $4.64 \pm 0.60$ & $<0.001$ & 1.000 & 0.144 & 0.066 \\
\hline$b=2000$ & $4.16 \pm 0.99$ & $4.10 \pm 1.00$ & $3.74 \pm 1.03$ & $<0.001$ & 1.000 & 0.002 & 0.001 \\
\hline \multicolumn{8}{|c|}{ Conspicuity of the left lobe } \\
\hline$b=800$ & $4.63 \pm 0.67$ & $4.56 \pm 0.71$ & $4.40 \pm 0.77$ & 0.001 & 1.000 & 0.370 & 0.131 \\
\hline$b=2000$ & $3.64 \pm 1.13$ & $3.45 \pm 1.18$ & $3.14 \pm 1.05$ & $<0.001$ & 0.268 & 0.008 & $<0.001$ \\
\hline \multicolumn{8}{|c|}{ Visibility of intrahepatic vessels } \\
\hline$b=800$ & $4.93 \pm 0.26$ & $4.70 \pm 0.54$ & $4.46 \pm 0.71$ & $<0.001$ & 0.190 & 0.109 & $<0.001$ \\
\hline$b=2000$ & $3.88 \pm 1.04$ & $3.68 \pm 1.11$ & $3.20 \pm 1.14$ & $<0.001$ & 0.246 & $<0.001$ & $<0.001$ \\
\hline \multicolumn{8}{|l|}{ Artefacts } \\
\hline$b=800$ & $4.95 \pm 0.22$ & $4.69 \pm 0.52$ & $4.06 \pm 0.75$ & $<0.001$ & 0.207 & $<0.001$ & $<0.001$ \\
\hline$b=2000$ & $4.70 \pm 0.62$ & $4.53 \pm 0.64$ & $3.49 \pm 0.73$ & $<0.001$ & 0.537 & $<0.001$ & $<0.001$ \\
\hline \multicolumn{8}{|c|}{ Overall image quality } \\
\hline$b=800$ & $4.80 \pm 0.40$ & $4.70 \pm 0.49$ & $4.13 \pm 0.75$ & $<0.001$ & 1.000 & $<0.001$ & $<0.001$ \\
\hline$b=2000$ & $4.13 \pm 0.82$ & $4.05 \pm 0.87$ & $3.40 \pm 0.69$ & $<0.001$ & 1.000 & $<0.001$ & $<0.001$ \\
\hline
\end{tabular}

Means and standard deviations of image quality obtained by two radiologists are summarized. $b 800, b=800 \mathrm{~s} / \mathrm{mm}^{2} ; \mathrm{b} 2000, \mathrm{~b}=2000 \mathrm{~s} / \mathrm{mm}^{2} ; \mathrm{C}-\mathrm{DWl}$, conventional diffusion-weighted imaging; SMS2-DWI, simultaneous multislice accelerated DWI with an acceleration factor of 2; SMS3-DWI, simultaneous multislice accelerated DWI with an acceleration factor of 3

Table 3 Results of the SNR analysis for the three sequences

\begin{tabular}{lccccccc}
\hline & C-DWI & SMS2-DWI & SMS3-DWI & $\begin{array}{l}\text { P values } \\
\text { Overall }\end{array}$ & $\begin{array}{l}\text { P values } \\
\text { C vs. SMS2 }\end{array}$ & $\begin{array}{l}\text { P values } \\
\text { SMS2 vs. SMS3 }\end{array}$ & $\begin{array}{l}\text { P values } \\
\text { C vs. SMS3 }\end{array}$ \\
\hline SNR & & & & & & & \\
$b=800$ & $107.55 \pm 48.56$ & $103.51 \pm 53.90$ & $75.22 \pm 57.60$ & 0.015 & 1.000 & 0.059 & 0.024 \\
$b=2000$ & $50.88 \pm 25.31$ & $47.30 \pm 23.11$ & $38.95 \pm 28.72$ & 0.110 & 1.000 & 0.452 & 0.123 \\
\hline
\end{tabular}

Means and standard deviations are shown for all sequences. SNR, signal-to-noise ratio; b800, b=800 s/mm²; b2000, b= $2000 \mathrm{~s} / \mathrm{mm}^{2}$; C-DWI, conventional diffusionweighted imaging; SMS2-DWI, simultaneous multislice accelerated DWI with an acceleration factor of 2; SMS3-DWI, simultaneous multislice accelerated DWI with an acceleration factor of 3
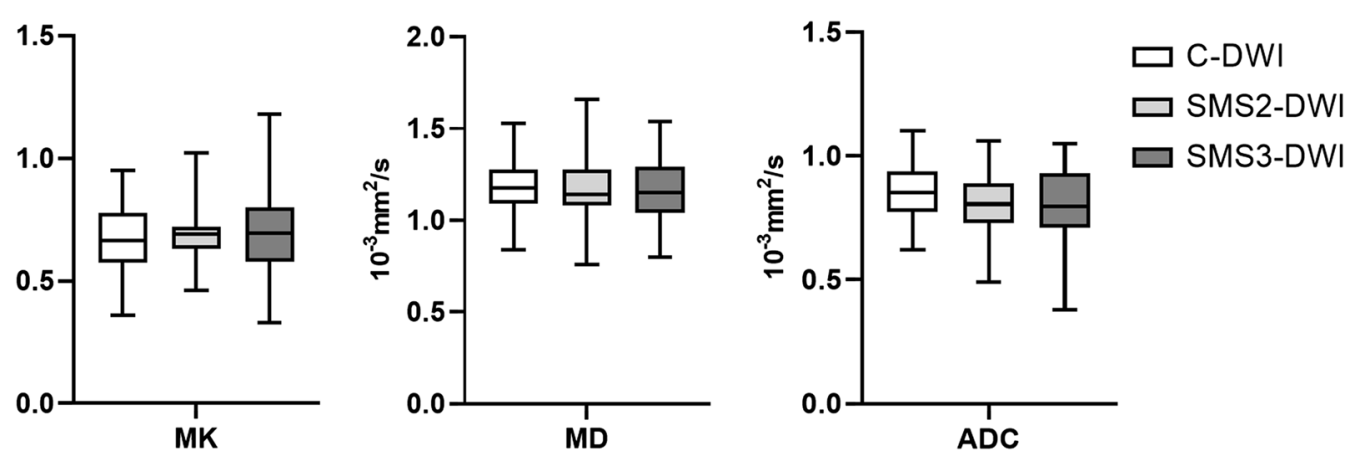

Fig. 3 Box-and-whisker plots for MK, MD and ADC values of the liver in C-DWI, SMS2-DWI and SMS3-DWI sequences. The bottom and top of the boxes indicate the min and max of the values, respectively. The horizontal line inside the box indicates median values. The MK values of C-DWI, SMS2-DWI and SMS3-DWI were $0.67 \pm 0.15,0.68 \pm 0.11$, and $0.70 \pm 0.18$, respectively. The MD values of C-DWI, SMS2-DWI and SMS3-DWI were $1.19 \pm 0.15 \times 10^{-3} \mathrm{~mm}^{2} / \mathrm{s}, 1.17 \pm 0.21 \times 10^{-3} \mathrm{~mm}^{2} / \mathrm{s}$, and $1.17 \pm 0.18 \times 10^{-3} \mathrm{~mm}^{2} / \mathrm{s}$, respectively. The ADC values of C-DWI, SMS2-DWI and SMS3-DWI were $0.85 \pm 1.12 \times 10^{-3} \mathrm{~mm}^{2} / \mathrm{s}, 0.81 \pm 1.24 \times 10^{-3} \mathrm{~mm}^{2} / \mathrm{s}$ and $0.80 \pm 1.56 \times 10^{-3} \mathrm{~mm}^{2} / \mathrm{s}$, respectively. There were no statistically significant differences between the three sequences (MK, $p=0.397 ; \mathrm{MD}, p=0.461 ; \mathrm{ADC}, p=1.346$ ). With an increase in the acceleration factors, MK values showed an increasing trend and MD and ADC values showed a decreasing trend in the liver parenchyma 


\section{Discussion}

In the present study, SMS-DWI with an acceleration factor of 2 obviously decreased the scan time without a negative impact on the image quality and maintained comparable DKI parameters and ADC values compared with those of C-DWI. Although the scan time was significantly shortened for SMS3-DWI sequences, it had a negative impact on the image quality, especially at a high b-value of $2000 \mathrm{~s} / \mathrm{mm}^{2}$.

The DKI model potentially reflects the non-Gaussian diffusion behaviour of water diffusivity in tissues using ultrahigh b-values above $1000 \mathrm{~s} / \mathrm{mm}^{2}\left(1500-2000 \mathrm{~s} / \mathrm{mm}^{2}\right.$ in body imaging) $[5,6,18]$; thus, DKI could provide further information on tissue characteristics and has additional value in the prediction of microvascular invasion
(MVI) of hepatocellular carcinoma (HCC) and in the assessment of post-therapeutic response in hypervascular HCC [3-5]. Obtaining ultrahigh b-value DWI images will induce a decreased SNR, increased distortion, susceptibility artefacts, and increased scan time [19].

In conventional diffusion acquisition, a single slice is excited, whereas in SMS-DWI, multiple slices are excited simultaneously, and the corresponding read-out contains these multiple slices. This permits the TR to be decreased, thus shortening the acquisition time, or allows more slices with thinner slice thicknesses to be scanned under the same TR $[7,9,10]$. Notably, the maximum number of simultaneous acquisition slices depends on the number of coil channels [7]. In theory, image acquisition in SMS-DWI can be accelerated to a large extent by
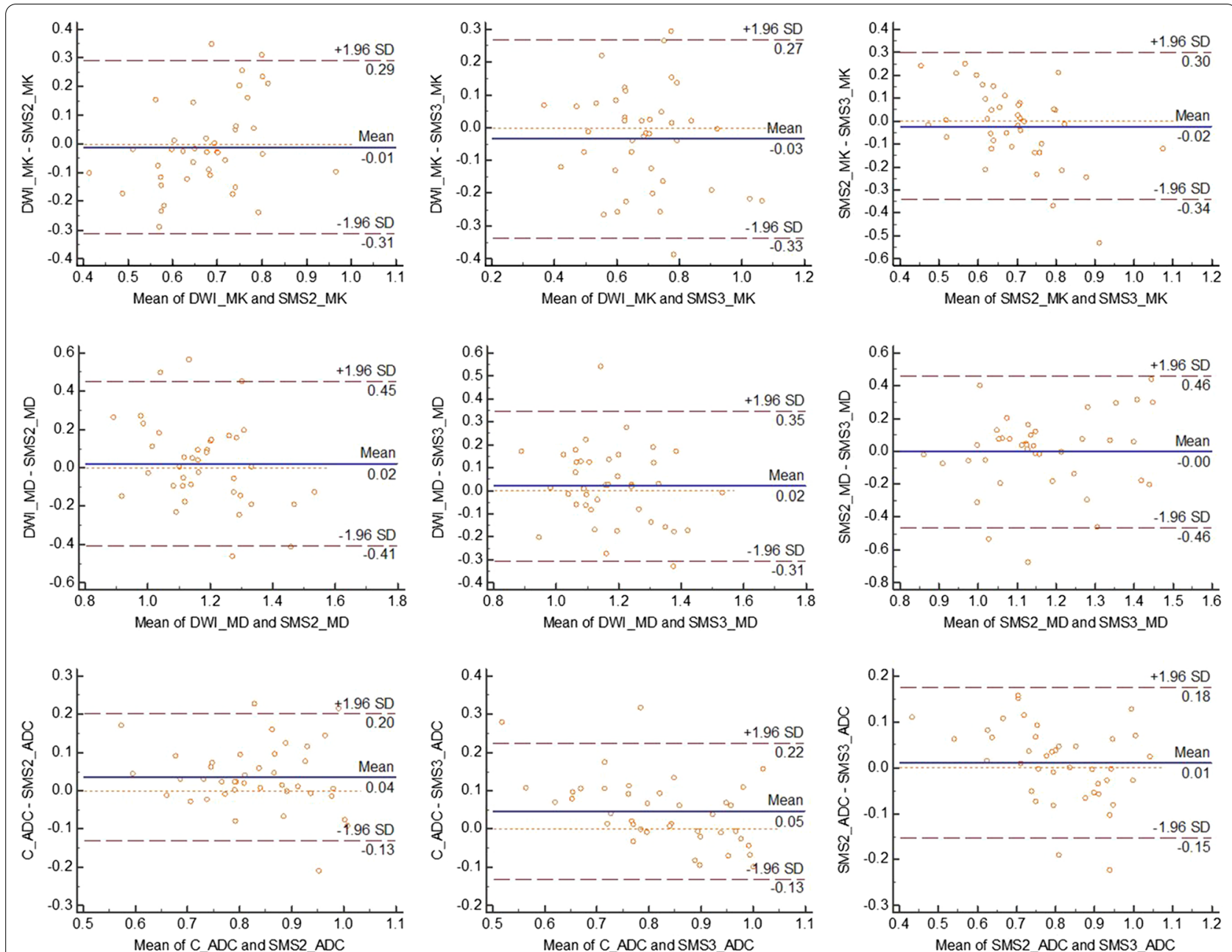

Fig. 4 Bland-Altman plots comparing DKI parameters and ADC values between the three sequences. The first line represents MK values, the second line represents MD values, and the third line represents ADC values. The MD and ADC values are given in $10^{-3} \mathrm{~mm}^{2} / \mathrm{s}$. The solid line indicates the mean absolute difference between two sequences, and the dashed lines indicate the $95 \%$ confidence interval of the mean difference. SD standard deviation 
increasing the applied acceleration factors. However, an arbitrary increase in the acceleration factors is expected to be associated with a decrease in image quality.

Our research indicates that SMS can shorten the scan time without reducing the image quality even in images with ultrahigh b-values of $2000 \mathrm{~s} / \mathrm{mm}^{2}$ if an acceleration factor of 2 is used. Although there were no statistically significant differences in the DKI parameters between SMS-DWI and C-DWI in this study, SMS3-DWI should not be chosen in future studies due to the obviously decreased image quality and pronounced artefacts, especially in images with high b-values of $2000 \mathrm{~s} / \mathrm{mm}^{2}$.

The MK values (unitless) of the DKI parameters reflect complex tissue microenvironments, such as tumour cells, necrosis, and inflammation. The MD values are the diffusion coefficients (unit: $10^{-3} \mathrm{~mm}^{2} / \mathrm{s}$ ) related to Gaussian behaviour and similar to the ADC values that decrease with restricted diffusion. MK is determined by the SI decay curvature away from the plot that would be predicted by a monoexponential fit, whereas MD is determined by the slope of the SI decay plot as b approaches 0 . Decreases in MD values and increases in MK values indicate abnormal diffusion behaviour in the organs. Although there were no statistically significant differences between the three sequences, with an increase in the acceleration factors, MK values showed an increasing trend and MD and ADC values showed a decreasing trend in the liver. This result could be due to a decrease in image quality that potentially affects the signal accuracies and influences the DKI parameter calculations in the SMS-DWI sequences.

Several studies have indicated that ADC values decrease when using SMS-DWI compared with C-DWI in the liver, pancreas and kidney [12, 15, 20]. One possible reason for the lower ADC values with higher acceleration factors is the shorter repetition time (TR) of the SMS sequence. In the present study, the TR was 5200, 2400, and $1800 \mathrm{~ms}$ for C-DWI, SMS2-DWI, and SMS3DWI, respectively. The lower TR may lead to a reduced signal due to T1 saturation effects, particularly with the high b-value images that had higher AFs, resulting in decreased ADC values.

Obele et al., who investigated the SMS technique with an acceleration factor of 2 in free breathing, noted that there were slightly lower ADC values compared with those of a conventional DWI sequence without statistical significance in the liver [12]. Another reason may be that we scanned the $3 \mathrm{~b}$-values together, which induced an increase in TE and led to a lower SNR in the $2000 \mathrm{~s} / \mathrm{mm}^{2} \mathrm{~b}$-value images, which also influenced the ADC values to some degree. When the ADC value was used for diagnosis in the liver, there were possibly lower ADC values in SMS sequences. Considering scan time, image quality and ADC values, an acceleration factor of 2 would be the best option for a clinical workflow in the liver.

There are several limitations of the study. First, the SMS-DWI and C-DWI sequences were scanned during free breathing and without respiratory-triggered techniques in our study. Respiratory motion and hepatic local motion may impact the image quality and challenge the reliability of the computation of DKI parameters to some degree. Several studies have indicated that compared with conventional DWI, SMS-DWI combined with respiratory triggering could improve image quality in the liver and kidney [21-23]. SMS-DWI sequences with respiratory compensation techniques should be implemented in the future.

Second, a comparison and assessment of DKI parameters using SMS-DWI were performed in relatively young and healthy participants. The diffusion coefficients can differ in normal tissues, diseased tissues, and focal lesions. Thus, the DKI parameters based on SMS-DWI for evaluating diffusive diseases, such as liver fibrosis, cirrhosis, and focal lesions, need to be further studied.

\section{Conclusion}

In conclusion, compared with conventional DWI sequences, SMS-DWI using an acceleration factor of 2 could reduce the scan time significantly and without negative effects on image quality and comparable DKI parameters and ADC values. Based on the present study results, SMS-DWI can promote DKI sequences in clinical settings. Further studies are needed to investigate the clinical diagnostic performance of DKI based on the SMS technique for evaluating diffuse diseases and focal lesions of the liver.

\section{Abbreviations}

DKI: Diffusion kurtosis imaging; SMS: Simultaneous multislice; MK: Apparent mean kurtosis; MD: Apparent mean diffusivity; ADC: Apparent diffusion coefficient; SNR: Signal-to-noise ratio; ROI: Region of interest.

\section{Authors' contributions}

$\mathrm{HX}$ and ZHY designed this study. HX collected patient data and was a major contributor to writing the manuscript. DWY and AhR analysed and evaluated the three types of sequential images. NZ, QZ, and HR contributed to the

acquisition of data and the analysis and interpretation of the data. JXZ and GJL provided guidance on magnetic resonance techniques. All authors read and approved the final manuscript.

\section{Funding}

This work is supported by the National Natural Science Foundation of China (No. 61871276) and Capital's Funds for Health Improvement and Research (No. 2018-2-2023).

Availability of data and materials

The datasets used and analysed in the current study are available from the corresponding author on reasonable request 


\section{Declarations}

\section{Ethics approval and consent to participate}

The study was approved by the Review Committee of Beijing Friendship Hospital, Capital Medical University. All methods were carried out in accordance with relevant guidelines and regulations, and written informed consent was obtained from all participants.

\section{Consent for publication}

Written informed consent for publication was obtained from all participants.

\section{Competing interests}

The authors declare that they have no competing interests.

\section{Author details}

'Department of Radiology, Beijing Friendship Hospital, Capital Medical University, 95 Yong an Road, Xicheng District, Beijing 100050, China. ${ }^{2}$ Clinical Epidemiology and EBM Unit, Beijing Friendship Hospital, Capital Medical University, Beijing 100050, China. ${ }^{3}$ MR Collaboration, Siemens Healthineers Ltd., Beijing, China. ${ }^{4}$ MR Application, Siemens Healthineers Ltd., Guangzhou, China.

Received: 31 March 2021 Accepted: 28 August 2021

Published online: 09 September 2021

\section{References}

1. Jensen JH, Helpern JA, Ramani A, Lu H, Kaczynski K. Diffusional kurtosis imaging: the quantification of non-gaussian water diffusion by means of magnetic resonance imaging. Magn Reson Med. 2005;53(6):1432-40.

2. Yang $L$, Rao S, Wang W, Chen C, Ding Y, Yang C, Grimm R, Yan X, Fu C, Zeng M. Staging liver fibrosis with DWI: is there an added value for diffusion kurtosis imaging? Eur Radiol. 2018;28(7):3041-9.

3. Goshima S, Kanematsu M, Noda Y, Kondo H, Watanabe H, Bae KT. Diffusion kurtosis imaging to assess response to treatment in hypervascular hepatocellular carcinoma. AJR Am J Roentgenol. 2015;204(5):W543-549.

4. Wang WT, Yang L, Yang ZX, Hu XX, Ding Y, Yan X, Fu CX, Grimm R, Zeng MS, Rao SX. Assessment of microvascular invasion of hepatocellular carcinoma with diffusion kurtosis imaging. Radiology. 2018:286(2):571-80.

5. Rosenkrantz AB, Padhani AR, Chenevert TL, Koh DM, De Keyzer F, Taouli B, Le Bihan D. Body diffusion kurtosis imaging: basic principles, applications, and considerations for clinical practice. J Magn Reson Imaging. 2015;42(5):1190-202.

6. Pasicz K, Podgorska J, Jasieniak J, Fabiszewska E, Skrzynski W, Anysz-Grodzicka A, Cieszanowski A, Kukolowicz P, Grabska I. Optimal b-values for diffusion kurtosis imaging of the liver and pancreas in MR examinations. Phys Med. 2019;66:119-23.

7. Barth M, Breuer F, Koopmans PJ, Norris DG, Poser BA. Simultaneous multislice (SMS) imaging techniques. Magn Reson Med. 2016;75(1):63-81.

8. Fritz J, Fritz B, Zhang J, Thawait GK, Joshi DH, Pan L, Wang D. Simultaneous multislice accelerated turbo spin echo magnetic resonance imaging: comparison and combination with in-plane parallel imaging acceleration for high-resolution magnetic resonance imaging of the knee. Invest Radiol. 2017;52(9):529-37.

9. Setsompop K, Cohen-Adad J, Gagoski BA, Raij T, Yendiki A, Keil B, Wedeen VJ, Wald LL. Improving diffusion MRI using simultaneous multi-slice echo planar imaging. Neuroimage. 2012;63(1):569-80.

10. Setsompop K, Gagoski BA, Polimeni JR, Witzel T, Wedeen VJ, Wald LL. Blipped-controlled aliasing in parallel imaging for simultaneous multislice echo planar imaging with reduced g-factor penalty. Magn Reson Med. 2012;67(5):1210-24.

11. Van Essen DC, Ugurbil K, Auerbach E, Barch D, Behrens TE, Bucholz R, Chang A, Chen L, Corbetta M, Curtiss SW, et al. The Human Connectome Project: a data acquisition perspective. Neuroimage. 2012;62(4):2222-31.

12. Obele CC, Glielmi C, Ream J, Doshi A, Campbell N, Zhang HC, Babb I. Bhat $\mathrm{H}$, Chandarana $\mathrm{H}$. Simultaneous multislice accelerated freebreathing diffusion-weighted imaging of the liver at 3T. Abdom Imaging. 2015:40(7):2323-30.

13. Boss A, Barth B, Filli L, Kenkel D, Wurnig MC, Piccirelli M, Reiner CS. Simultaneous multi-slice echo planar diffusion weighted imaging of the liver and the pancreas: optimization of signal-to-noise ratio and acquisition time and application to intravoxel incoherent motion analysis. Eur J Radiol. 2016:85(11):1948-55

14. OhImeyer S, Laun FB, Palm T, Janka R, Weiland E, Uder M, Wenkel E. Simultaneous multislice echo planar imaging for accelerated diffusionweighted imaging of malignant and benign breast lesions. Invest Radiol. 2019:54:524-30.

15. Kenkel D, Barth BK, Piccirelli M, Filli L, Finkenstadt T, Reiner CS, Boss A. Simultaneous multislice diffusion-weighted imaging of the kidney: a systematic analysis of image quality. Invest Radiol. 2017;52(3):163-9.

16. Park JH, Seo N, Lim JS, Hahm J, Kim MJ. Feasibility of simultaneous multislice acceleration technique in diffusion-weighted magnetic resonance imaging of the rectum. Korean J Radiol. 2020;21(1):77-87.

17. Van Phi VD, Becker AS, Ciritsis A, Reiner CS, Boss A. Intravoxel incoherent motion analysis of abdominal organs: application of simultaneous multislice acquisition. Invest Radiol. 2018;53(3):179-85.

18. Kuai ZX, Sang XQ, Yao YF, Chu CY, Zhu YM. Evaluation of non-monoexponential diffusion models for hepatocellular carcinoma using b values up to $2000 \mathrm{~s} / \mathrm{mm}(2)$ : a short-term repeatability study. J Magn Reson Imaging. 2019:50(1):297-304.

19. Dreher C, Kuder TA, Konig F, Paech D, Tavakoli A, Laun FB, Flothow F, Gnirs $\mathrm{R}$, Benkert T, Strecker R, et al. Advanced diffusion-weighted abdominal imaging: qualitative and quantitative comparison of high and ultrahigh b-values for lesion detection and image quality. Invest Radiol. 2020:55(5):285-92.

20. Taron J, Martirosian P, Kuestner T, Schwenzer NF, Othman A, Weiss J, Notohamiprodjo M, Nikolaou K, Schraml C. Scan time reduction in diffusionweighted imaging of the pancreas using a simultaneous multislice technique with different acceleration factors: how fast can we go? Eur Radiol 2018;28(4):1504-11.

21. Taron J, Martirosian P, Erb M, Kuestner T, Schwenzer NF, Schmidt H, Honndorf VS, Weibeta J, Notohamiprodjo M, Nikolaou K, et al. Simultaneous multislice diffusion-weighted MRI of the liver: analysis of different breathing schemes in comparison to standard sequences. J Magn Reson Imaging. 2016;44(4):865-79.

22. Tavakoli A, Attenberger UI, Budjan J, Stemmer A, Nickel D, Kannengiesser S, Morelli JN, Schoenberg SO, Riffel P. Improved liver diffusion-weighted imaging at $3 \mathrm{~T}$ using respiratory triggering in combination with simultaneous multislice acceleration. Invest Radiol. 2019;54(12):744-51.

23. Tavakoli A, Krammer J, Attenberger UI, Budjan J, Stemmer A, Nickel D, Kannengiesser S, Morelli JN, Schoenberg SO, Riffel P. Simultaneous multislice diffusion-weighted imaging of the kidneys at 3 T. Invest Radiol. 2020;55:233-8.

\section{Publisher's Note}

Springer Nature remains neutral with regard to jurisdictional claims in published maps and institutional affiliations. 